\title{
Os sessenta anos do Teatro Experimental do Porto (1953-2013)
}

\author{
Rui Pina Coelho'
}

[0] principal objectivo do teatro experimental é tornar-se desnecessário, isto é - elevar o gosto do público e o nível estético do teatro. António Pedro (1957: 23)

No pós-Segunda Guerra Mundial, depois da derrota do eixo italo-germânico, em Portugal assiste-se a um abrandar dos processos censórios impostos pelo regime fascista. É nesse contexto que surgirão diversos colectivos que se encarregarão de dar corpo a um dos momentos mais excitantes do teatro em Portugal. São grupos como o Teatro-Estúdio do Salitre (1946), a Casa da Comédia (1946), os Companheiros do Páteo das Comédias (1948), o Grupo Dramático Lisbonense (1948-50), o Grupo de Teatro Experimental - da Rua da Fé (1951), o Teatro d'Arte de Lisboa (1955) e, claro está, o Teatro Experimental do Porto.

Quanto a este último, foi desde 1950 que "um grupo de pessoas preocupadas com os problemas da cultura em geral, e muito especialmente do teatro, animadas pelos ventos de Lisboa e seguindo o exemplo do que acontecera em algumas cidades europeias, resolveu fundar um grupo de teatro" (AA.W. 1982: 9). A aprovação dos seus estatutos acontecerá somente em 1951 e a sua legalização em 1952. Dentro das primeiras actividades contam-se uma conferência promovida por Hernâni Silva no Instituto Francês do Porto e o início dos ensaios de Antígona de Jean Anouilh.

Não obstante o seu fulgor inicial, o mo(vi)mento de teatro de todos aqueles grupos experimentais será de curta duração e, por meados da década de cinquenta, todos terão desaparecido. Todos excepto um: o Círculo de Cultura Teatral - Teatro Experimental do Porto. Assim, por toda a década de cinquenta, será o TEP - o solitário sobrevivente - a transportar a mecha do experimentalismo (sinónimo, neste contexto, de uma atitude de divulgação de obras e autores, na valorização de uma ética em relação ao trabalho teatral, na rejeição dos interesses comerciais e das convenções da profissão). Será este experimentalismo que alimentará a constituição do movimento de teatro independente por meados da década de sessenta e setenta em Portugal.

Nos seus primeiros anos de vida, a história do TEP confunde-se com a de António Pedro, um dos mais respeitados encenadores e pensadores sobre a matéria teatral do seu tempo. A. Pedro assume a direcção do TEP a 7 de Março de 1953, deixando-a em Fevereiro de 1961. Nos quase dez anos em que esteve à frente da companhia, transformou a paisagem cultural da cidade e do pais, levando à cena um repertório exigente e informado, nada consentâneo com a mediania geral vigente no teatro seu contemporâneo: Shakespeare, Ben Jonson, Kleist, Ibsen, Tchekov, Synge, Miller, O'Neill, lonesco, António José da Silva, Bernardo Santareno, são alguns exemplos. Criou igualmente as bases para uma verdadeira escola de actores (de onde sairão artistas como Dalila Rocha, João Guedes, Baptista Fernandes, Mário Jacques, Vasco de Lima Couto, Alda Rodrigues, Jaime Valverde, Sinde Filipe, Fernanda Gonçalves, José Silva, José Pina, José Brás, Madalena Braga...), concebeu a companhia como um espaço privilegiado de experimentação ao nível da cenografia, da iluminação, da sonoplastia e dos figurinos e, muito significativamente, consolidou a prática da encenação em Portugal operando uma das mais relevantes renovações cénicas no teatro do século XX português.

0 TEP, amador nos primeiros anos de existência e profissional a partir de 1957, surge no enquadramento de um Círculo de Cultura Teatral (CCT), cujos fins, explicitados no artigo $2^{\circ}$ dos seus estatutos, são, entre outros, "realizar uma obra de cultura teatral, alheia a quaisquer lucros materiais e a fins políticos ou religiosos", mas também "elevar o nivel moral, intelectual e artístico do Teatro, promovendo a representação das melhores obras nacionais e estrangeiras e estimulando os seus autores, artistas e técnicos" e "criar e manter um Grupo de Teatro Experimental que terá como objectivo a realização de experiências de encenação, de representação e de decoração" Estatutos do Círculo de Cultura Teatral - Grupo de Teatro Experimental do Porto, aprovados pelo alvará no 45 do Governo Civil do Porto, 21 de Outubro de 1952. Porto: Imprensa Social, 1957, p. $5 .$.

A ambição das suas propostas iniciais não se consumirá. Ao longo destes sessenta anos, desenha-se 0 perfil de um colectivo de teatro de arte, vocacionado para um teatro popular, militante e preocupado. Desde a estreia das três peças curtas - A gota de mel, de Léon Chancerel, Nau catrineta, de Egito Gonçalves, e Um pedido de casamento, de Anton Tchekov - que constituíram o primeiro espectáculo, com estreia a 18 de Junho de 1953 até à estreia de As relações de Clara, de Dea Loher, em Novembro de 2013, o 233 espectáculo da companhia, o TEP assegurou um repertório de espantosa pertinência. Foram espectáculos difíceis, premiados, polémicos; comédias ligeiras, tragédias, dramas e farsas; os clássicos
Para a organização deste Portefólio, muito agradeço a inestimável ajuda de Júlio Gago, o actual Director do CCTTEP, e da secretária Ana Santos. 
e os contemporâneos; espectáculos para a infância e para o público escolar; foram publicações ensaísticas e de textos dramáticos; foi o cultivo de uma relação electiva com artistas plásticos... Foi, em suma, a criação de um património cultural - pela mão de cerca de mil e quinhentos artistas, técnicos e administrativos ao longo destes sessenta anos - de inestimável valor e que é uma porta aberta para a história do teatro em Portugal e na Europa. Uma longevidade e relevância que as seguintes fotografias de espectáculos deixam tímido - e insuficiente - traço.

\section{Referências bibliográficas}

AA.W. (1982) Circulo de leitura teatral / Teatro Experimental do Porto: 1952-1982 - trinta anos ao serviço da cultura e do teatro. Catálogo da exposição retrospectiva dos cinquenta primeiros espectáculos do TEP, Port, CCT/TEP

PEDRO, António (1975), O Teatro Experimental do Porto, separata da revista Lusiada, vo. III, n. ${ }^{\circ} 10$.

PORTO, Carlos (1997), O TEP e o teatro português: Histórias e imagens, Porto, Fundação Engenheiro António de Almeida.

Legendas

1> Um pedido de casamento, de Anton Tchekov, enc. António Pedro, 1953 (João Guedes, Correia Alves e Dalila Rocha), fot. José Mesquita.

2> A nau catrineta, de Egito Gonçalves,

enc. António Pedro, 1953 (Dalila Rocha, Natércia Pimentel, Manuela Delgado, Júlia Babo, António Maria, Américo Pessoa, Alexandre Babo, Correia Alves e Amadeu Meireles), fot. José Mesquita.

3> Antigona, texto e enc. António Pedro, 195 (Luis Nascimento, Alfredo Calheiros, Correia Alves, Manuela Delgado, João Guedes e Dalila Rocha),

fot. arquivo CCT/TEP.

4> Morte de um caixeiro viajante, de Arthur Miller, enc. António Pedro, 1954 (Vasco de Lima Couto, Baptista Fernandes, João Guedes e Dalila Rocha), fot. arquivo CCT/TEP.

5> Guerras do alecrim e manjerona

de António José da Silva, enc. António Pedro, 1956 (Jaime Valverde, João Guedes e Vasco de Lima Couto), fot. Fernando Aroso.

6> O landau de seis cavalos, de Victor Ruiz Iriarte, enc. António Pedro, 1955 (Júlia Babo e Vasco de Lima Couto), fot. arquivo CCT-TEP.

7> Macbeth, de Shakespeare, enc. António Pedro, 1956 (José Pina, João Guedes, Dalila Rocha, Baptista Fernandes, Fernando Gaspar e figuração), fot. José Mesquita.

8> Um deus dormiu lá em casa, de Guilherme Figueiredo, enc. Augusto Gomes, 1957 (Dalila Rocha, Inês Palma,

Vasco de Lima Couto e João Guedes), fot. Fernando Aroso. 9> 0 valentão do mundo ocidental, de J. M. Synge, enc António Pedro, 1957 (José Pina, Baptista Fernandes, João Guedes, Egito Gonçalves, Dalila Rocha e Vasco de Lima Couto), fot. Fernando Aroso.

10> A promessa, de Bernardo Santareno,

enc. António Pedro, 1957 (Alexandre Vieira, Dalila Rocha, João Guedes e Fernanda de Sousa), fot. Álvaro Portugal. 11> Jornada para a noite

de Eugene O'Neill, enc. António Pedro, 1958 (Alexandre Vieira, Baptista Fernandes, Dalila Rocha e João Guedes), fot. Fernando Aroso.

12> É urgente o amor, de Luiz Francisco Rebello, enc. António Pedro, 1958 (Cândida Lacerda, Dalila Rocha Baptista Fernandes e João Guedes), fot. Fernando Aroso.
13> Mar, de Miguel Torga, enc. António Pedro, 1958 (Dalila Rocha, Fernanda Gonçalves e Alda Rodrigues), fot. Fernando Aroso

14> Volpone, de Ben Jonson, enc. António Pedro, 1958 (João Guedes e Vasco de Lima Couto), fot. Fernando Aroso

15> Requiem, de William Faulkner, enc. António Pedro, 1958 (Dalila Rocha), fot. Fernando Aroso.

16> Quanto importa ser leal, de Oscar Wilde enc. António Pedro, 1960 (Dalila Rocha e Vasco de Lima Couto), fot. Andrelino Fernandes.

17> Hedda Gabler, de Henrik Ibsen, enc. João Guedes, 1961 (Nita Mercedes, Mário Jacques, Dalila Rocha e Vasco de Lima Couto), fot. Fernando Aroso.

18> 0 tio Vânia, de A. Tchekov, enc. João Guedes, 1960 (Vasco de Lima Couto e Dalila Rocha), fot. Fernando Aroso. 19> A mordaça, de Alfonse Sastre, enc. João Guedes, 1961 (Mário Jacques, Dalila Rocha e João Guedes) fot. Fernando Aroso.

20>0 vagabundo das mãos de oiro, de Romeu Correia, enc. João Guedes, 1962 (Nita Mercedes, José Brás Madalena Braga), fot. arquivo CCT/TEP.

21> Todos eram meus filhos, de Arthur Miller, enc. Rogério Paulo, 1963 (José Brás, Fernanda Gonçalves, Madalena Braga e Rogério Paulo), fot. Teófilo Rego. 22> A moratória, de Jorge Andrade,

enc. Alda Rodrigues, 1963 (Liliana de Abreu, Mário Jacques, Jaime Valverde e Alda Rodrigues),

fot. Teófilo Rego.

23> Os burossáurios, de Silvano Ambrogi,

enc. João Guedes, 1964 (Nunes Vidal, Rui Branco e António Montez), fot. Teófilo Rego.

24> A carta perdida, de Ion Luca Caragiale,

enc. Carlos Avilez, 1964 (Alda Rodrigues e António Montez), fot. Teófilo Rego.

25> Auto da Feira, de Gil Vicente, enc. Carlos Avilez, 1965 (Alina Vaz, Glicinia Quartim, Maria Emilia Correia e outros), fot. Teófilo Rego.

26> A grande cólera de Philippe Hotz, de Max Frisch, enc. João Guedes, 1965 (António Montez, Rui Branco,

Margarida Mauperrin e José Brás), fot. arquivo CCT-TEP.
27> 0 avançado centro morreu ao amanhecer

de Agustin Cuzanni, 1965 (António Pereira Manuel Gonçalves, Rui Branco e Alice Vasconcelos), fot. Horácio Rego.

28> 0 barbeiro de Sevilha, de Beaumarchais, enc. Glória de Matos e Francisco Russo, 1966 (Augusto Leal, figurante Luis Aires e Luis Alberto), fot. Horácio Rego. 29> 0 tempo e a ira, de John Osborne, enc. Fernando Gusmão, 1967 (Isabel de Castro e Luis Alberto), fot. Lúcio Estrela Santos. 30> 0 túnel, de Paër Lagerkvist, enc. João Guedes, 196 (José Brás e David Silva), fot. arquivo CCT/TEP.

31> Fim de festa, de Samuel Beckett, enc. Júlio Castronuovo, 1970 (Diamantino Silvestre, António Reis, Júlio Cardoso e Nita Mercedes),

fot. arquivo CCT/TEP. 32> Vestir os nus, de Luigi Pirandello,

enc. João Guedes, 1968 (Augusto Leal e Fernanda Alves), fot. arquivo CCT/TEP.

33> Mirandolina, de Goldoni, enc. Luis Tito, 1969 (Augusto Martins), fot. arquivo CCT/TEP.

34> Auto da Alma, de Gil Vicente, enc. Luis Tito, 1969 (Júlio Cardoso), fot. arquivo CCT/TEP.

35> A batalha naval, de Jaime Salazar Sampaio, enc. Carmen Gonzalez, 1971 (António Reis e Carmen Gonzalez), fot. arquivo CCT/TEP 36> A casa da Bernarda Alba, de Federico Garcia Lorca, enc. Angel Facio, 1972 (entre outros: Júlio Cardoso, Maria Emilia Correia, Márcia Breia, Manuela de Melo, Estrela Novais, Fernanda Gonçalves, Nita Mercedes, José Pinto e Alice Vasconcelos), fot. arquivo CCT/TEP. 37> Woyzeck, de Georg Büchner, enc. José Cayolla, 197 (Fernanda Gonçalves, Agostinho Dinis, José Pinto e Estrela Novais), fot. arquivo CCT/TEP. 38> Oh! Lusitânia Quão Bela Eras, de Roberto Merino / Peter Weiss, 1975 (João Enes e outros),

fot. arquivo CCT/TEP.

39> Schmürtz, de Boris Vian, enc. João Guedes, 1977 (João Paulo Costa, Laurinda Ferreira, Fernanda Gonçalves e José Pinto), fot. arquivo CCT/TEP. 
40> Os emigrantes, de Slawomir Mrozek, enc. João Lourenço, 1977 (António Montez e João Perry), fot. Fernando Gonçalves e Jorge Afonso. $41>$ As histórias de Hakim, de Norberto Ávila, enc. Jorge Pinto, 1978 (João Enes, Mário Sancho e Domingos Cerqueira), fot. arquivo CCT/TEP. 42> Yerma, Federico Garcia Lorca, enc. Xosé Blanco Gil, 1979 (Ana Bustorff, Marilia Gama, Fernanda Gonçalves e outros), fot. Xavier Neves.

43> Os preços voltam a atacar, de Jaime Salazar Sampaio, enc. José Cayolla, 1981 (Fernanda Gonçalves, João Enes e outros), fot. arquivo CCT/TEP. 44> Ubu, de Alfred Jarry, enc. Eduardo Freitas, 1981 (figurantes dos grupos Flor de Infesta e Presença), fot. arquivo CC/TEP.

45> A boda dos pequenos burgueses, de Bertolt Brecht, enc. Moncho Rodriguez, 1982 (Fernando Nascimento, Mário Sancho, Eugénia Bettencourt, José Pinto e João Cardoso), fot. arquivo CCT/TEP.

46> Eles não usam smoking, de Gianfranco Guarnieri, enc. Rogério Paulo, 1984 (Fernanda Gonçalves, Emilia Silvestre, José Pinto, José Moreira, Rogério Paulo, Xosé Maria Estraviz, Mário Sancho, Maria José Miranda, Isaura Melo e Jorge Pinto), fot. arquivo CCT/TEP.

47> Florânia ou a perfeita felicidade, de Norberto Ávila, enc. Moncho Rodriguez, 1983 (Rosa Quiroga, Emilia Silvestre, Fernanda Gonçalves, Mário Sancho, João Cardoso e Jorge Pinto), fot. arquivo CCT/TEP. 48> Amor de Dom Perlimplim com Belisa em seu jardim, de Federico Garcia Lorca, enc. Roberto Merino, 1985 (Emilia Silvestre e Isaura Melo), fot. arquivo CCT/TEP. 49> A birra do morto, de Vicente Sanchez, enc. Mário Viegas, 1986 (Mário Sancho, Nelinho e Fernando Sá), fot. arquivo CCT/TEP. 50> A catástrofe, de Samuel Beckett, enc. Mário Viegas, 1986 (Mário Viegas, Cândido Ferreira e José Moreira), fot. arquivo.

51> As histórias de Hakim, de Norberto Ávila, enc. Roberto Merino, 1988 (Margarida Machado e Alfredo Correia), fot. arquivo CCT/TEP. 52> Leôncio e Lena, de Georg Büchner, enc. Roberto Merino, 1989 (José Pinto), fot. arquivo CCT/TEP.
53> Max e Mila, de Volker Ludwig, enc. Júlia Correia 1990 (Pedro Aparicio, Margarida Machado e Jorge Vasques), fot. Úrsula Zanger.

54> Os cegos, de Michel Ghelderode,

enc. Cláudio Lucchesi, 1992 (Ângelo Neves, Tó Maia e Paulo Castro), fot. Carlos Guerra.

55> A paixão do jardineiro, de Jean Pierre Sarrazac,

enc. Fernando Mora Ramos, 1994 (Paulo Claro e Cecilia Guimarães), fot. Acácio de Carvalho.

56> Vanzetti, de Luis Araújo, enc. Acácio de Carvalho, 1995 (Ângela Marques e Gil Filipe), fot. Acácio de Carvalho. 57> Mauser, de Heiner Müller, enc. Paulo Castro, 1996 (José Pinto, João Pedro Vaz e João Cardoso), fot. Susana Paiva. 58> Comédia de bastidores, de Alan Ayckbourn, enc. João Cardoso, 1997 (Ângela Marques, Jorge Mota e Rosa Quiroga), fot. Pedro Morais.

59> A lenda de Gaia, de A. Garrett, enc. Norberto Barroca, 1999 (Manuela Martinez, Alexandra Ferreira, Norberto Barroca e José Brás), fot. José Martins.

$60>$ Tempo de festa, de Harold Pinter, enc. Júlia Correia, 2000 (Luis Campião e Joana Esteves), fot. José Martins. 61> Gaia d'Ouro, de Norberto Barroca e Manuel Dias, enc. Norberto Barroca, 2001 (Silvia Magalhães, Joana Esteves, Patricia Franco, Mariana Assunção, Diana Sá, Aquiles Dias, Ricardo Simões, Manuel Vieira, Adriano Martins, Luis Gonzaga, Oliveira Alves, David Cardodo, Silva Vilela e João Miguel Mota), fot. José Martins. 62> 0 café, de Fassbinder, enc. Rui Silva, 2002 (João Miguel Mota, Augusto Martins e Luis Gonzaga), fot. José Martins.

63> Teresa, de Émile Zola, enc. Norberto Barroca, 2002 (João Miguel Mota, Alice Vasconcelos, Augusto Martins, José Brás e Luis Gonzaga), fot. José Martins.

64> Antigona, de António Pedro, enc. Norberto Barroca, 2003 (Norberto Barroca e Alice Vasconcelos), fot. José Martins. 65> Os Maias, de Eça de Queirós, enc. Norberto Barroca, 2004 (João Miguel Mota e Paulo Narciso), fot. José Martins. 66> A cantora careca, de lonesco, enc. Norberto Barroca, 2004 (José Dias, Olga Dias, Aquiles Dias, João Miguel Mota e Susana Sá), fot. José Martins.
67> Separações, de Jean Cocteau, enc. Norberto Barroca, 2005 (Ângela Marques e José Dias), fot. José Martins. 68> 0 nosso hóspede, de Joe Orton enc. Norberto Barroca, 2006 (Daniel Alves, Olga Dias e José Dias), fot. José Martins. 60> João Gabriel Borkman, de lbsen, enc. Norberto Barroca, 2007 (Ricardo Trêpa, Henriqueta Maia, Olga Dias e Norberto Barroca), fot. José Martins. 70> Restos, de Bernardo Santareno, enc. José Dias, 2008 (Mané Carvalho e Daniel Pinto), fot. José Martins.

71> As espertezas de Figaro,

adapt. e enc. de Norberto Barroca, a partir de O barbeiro de Sevilha, de Beaumarchais, 2009 (Isabel Queirós, João Leiria, José Cruz, Luis Trigo e José Dias), fot. José Martins. 72> A morte de um caixeiro viajante, de Arthur Miller, enc. Gonçalo Amorim, 2010 (Cláudio da Silva e Maria João Pinho), fot. João Almeida.

73> Jornada para a noite, de Eugene O'Neill, enc. Nuno Cardoso, 2010 (Eduardo Breda, Ângela Marques e João Pedro Vaz), fot. João Pádua.

74> Felizmente há luar! de Luis de Sttau Monteiro, enc. Cláudio da Silva, 2011 (de frente: Ruben Andrade, Andrea Moisés, Ana Chagas, Aquiles Dias, Nuno Martins e José Brás; de costas: Andreas Dyrdal, Joel Sines e Tiago Sines), fot. José Martins 75> Os assassinos, de Miguel Castro Caldas, enc. Bruno Bravo, 2011 (Miguel Loureiro, Ricardo NevesNeves, Paulo Pinto e Dinis Gomes), fot. José Martins. 76> 0 dia do santo, de John Whiting, enc. Gonçalo Amorim, 2012 (Crista Alfaiate, Ana Brandão, Gonçalo Amorim e Nicolas Brites), fot. José Martins.

77> Chove em Barcelona, de Pau Miró, enc. Gonçalo Amorim, 2012 (Romeu Costa e Maria João Pinho), fot. José Martins. 78> Duas senhorinhas rumo ao Norte, de Pierre Notte, enc. Gonçalo Amorim, 2013 (Carla Miranda e Maria do Céu Ribeiro), fot. Paulo Pimenta. 


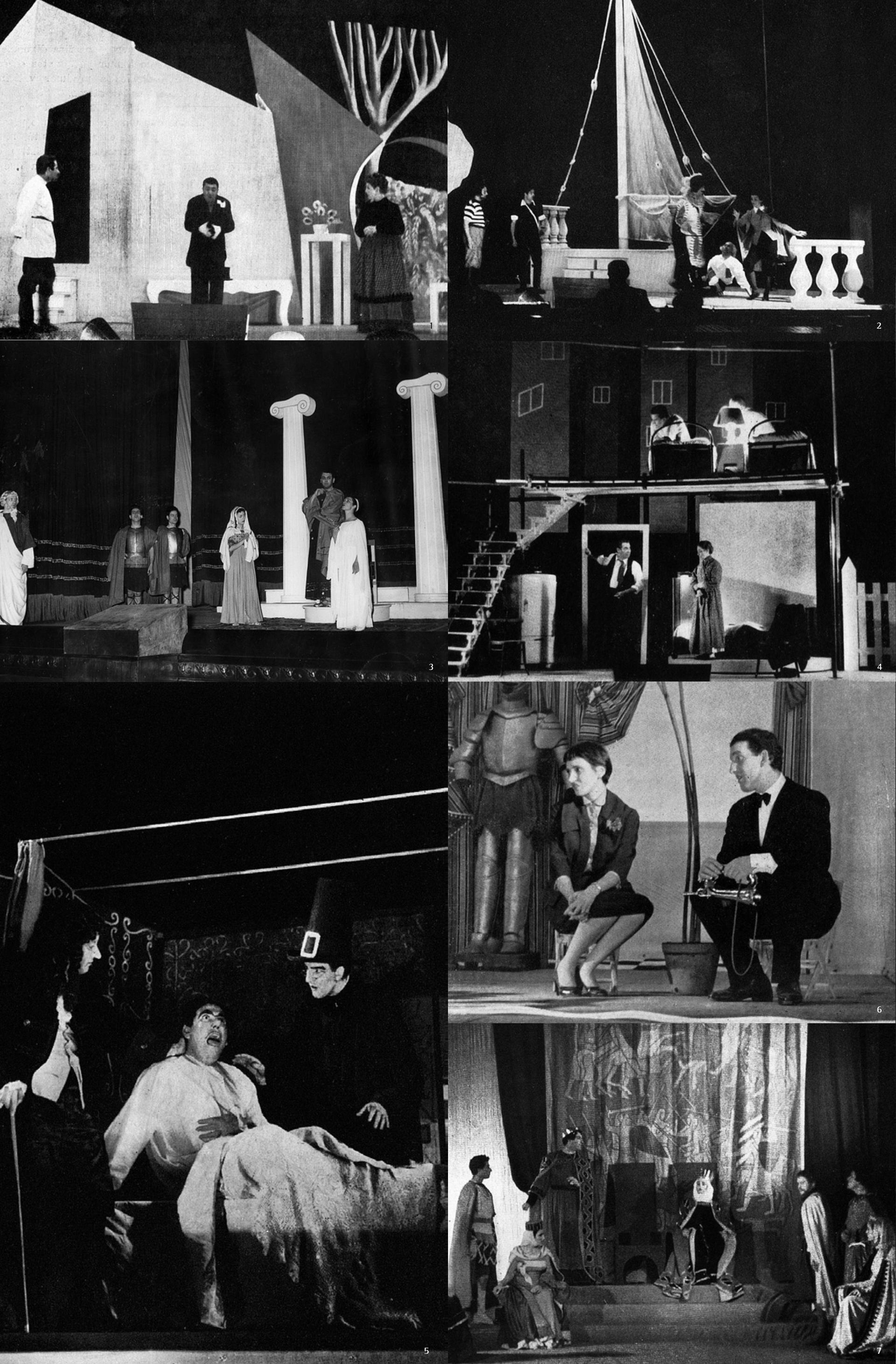




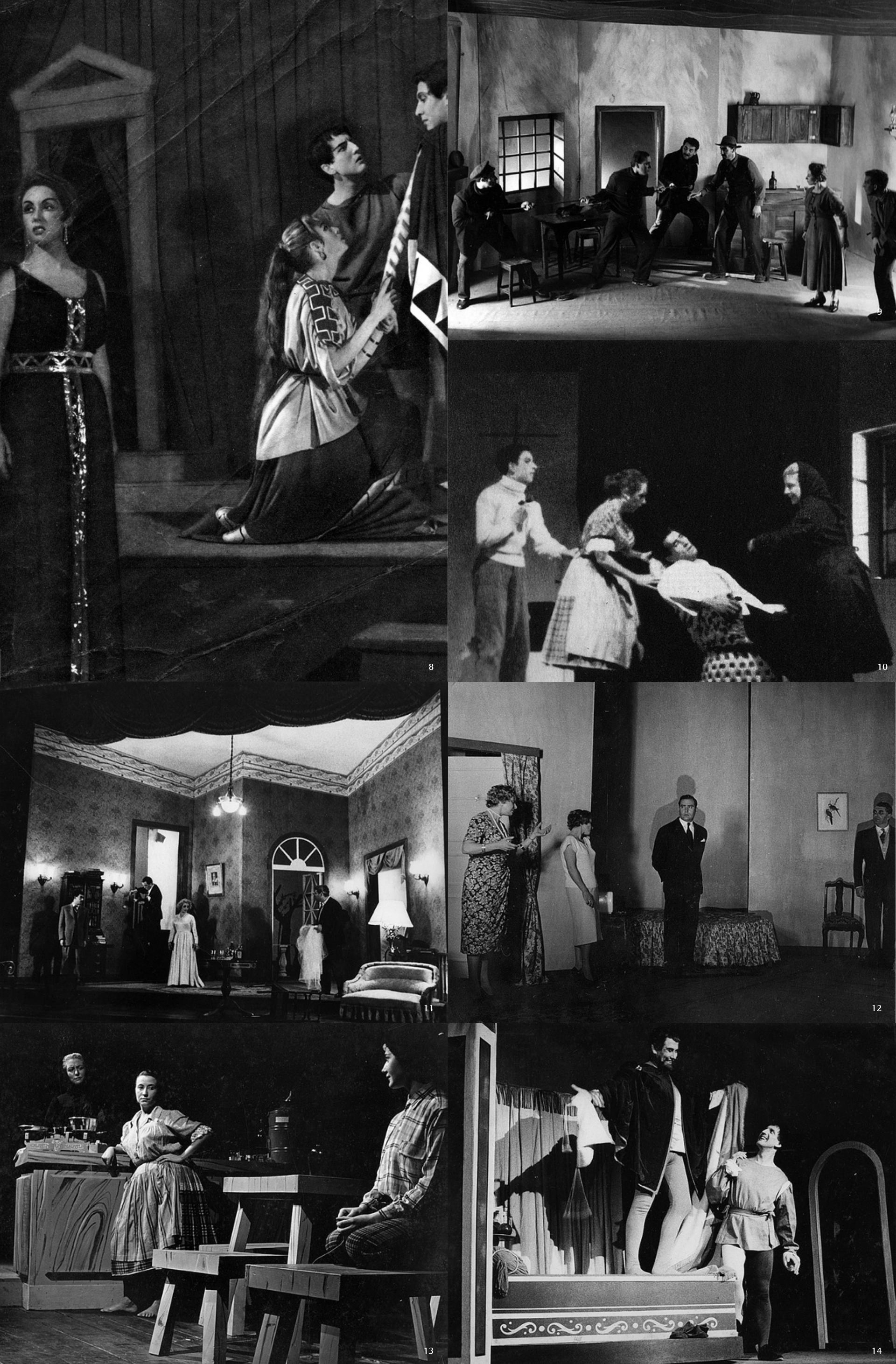



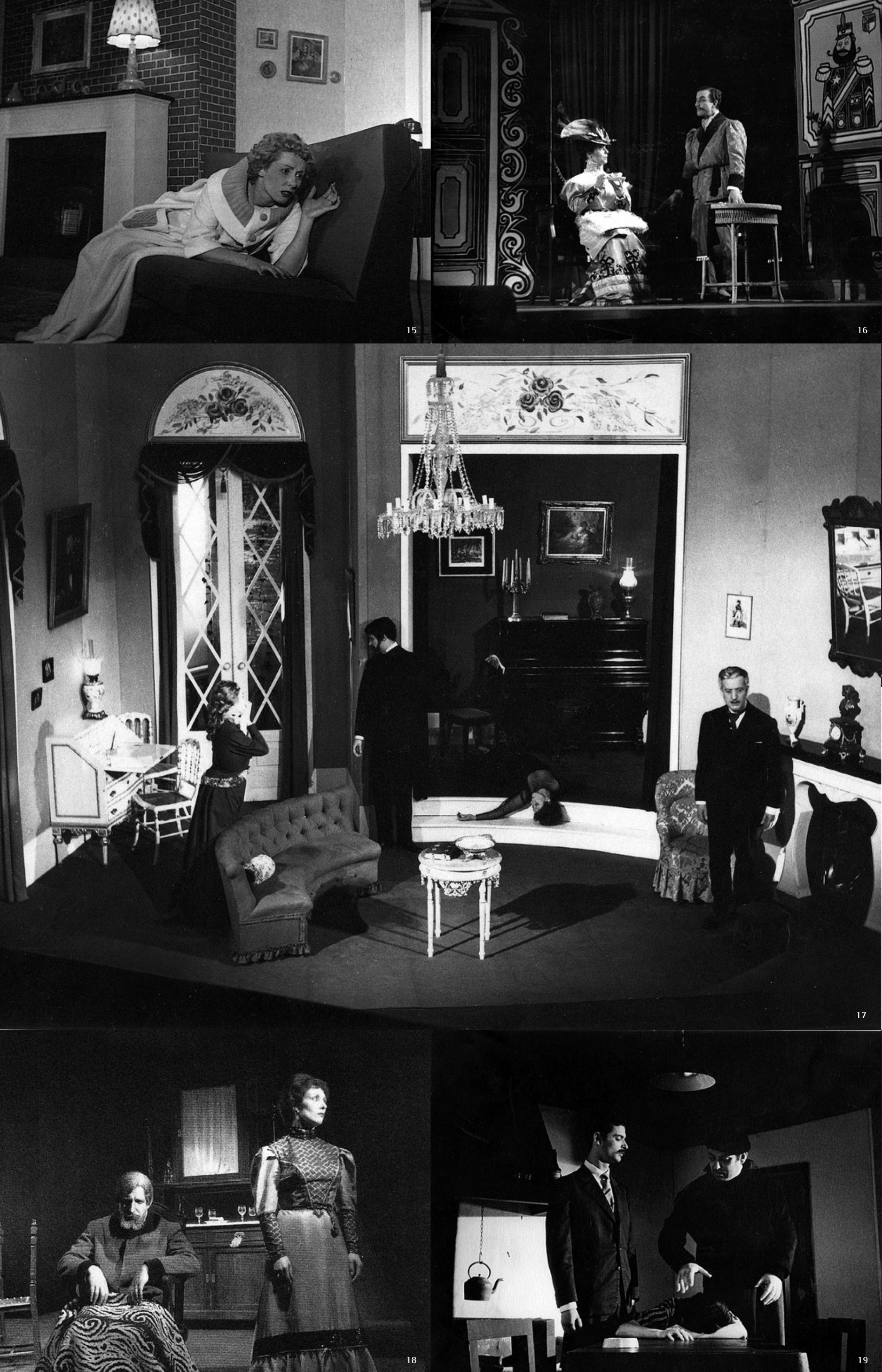


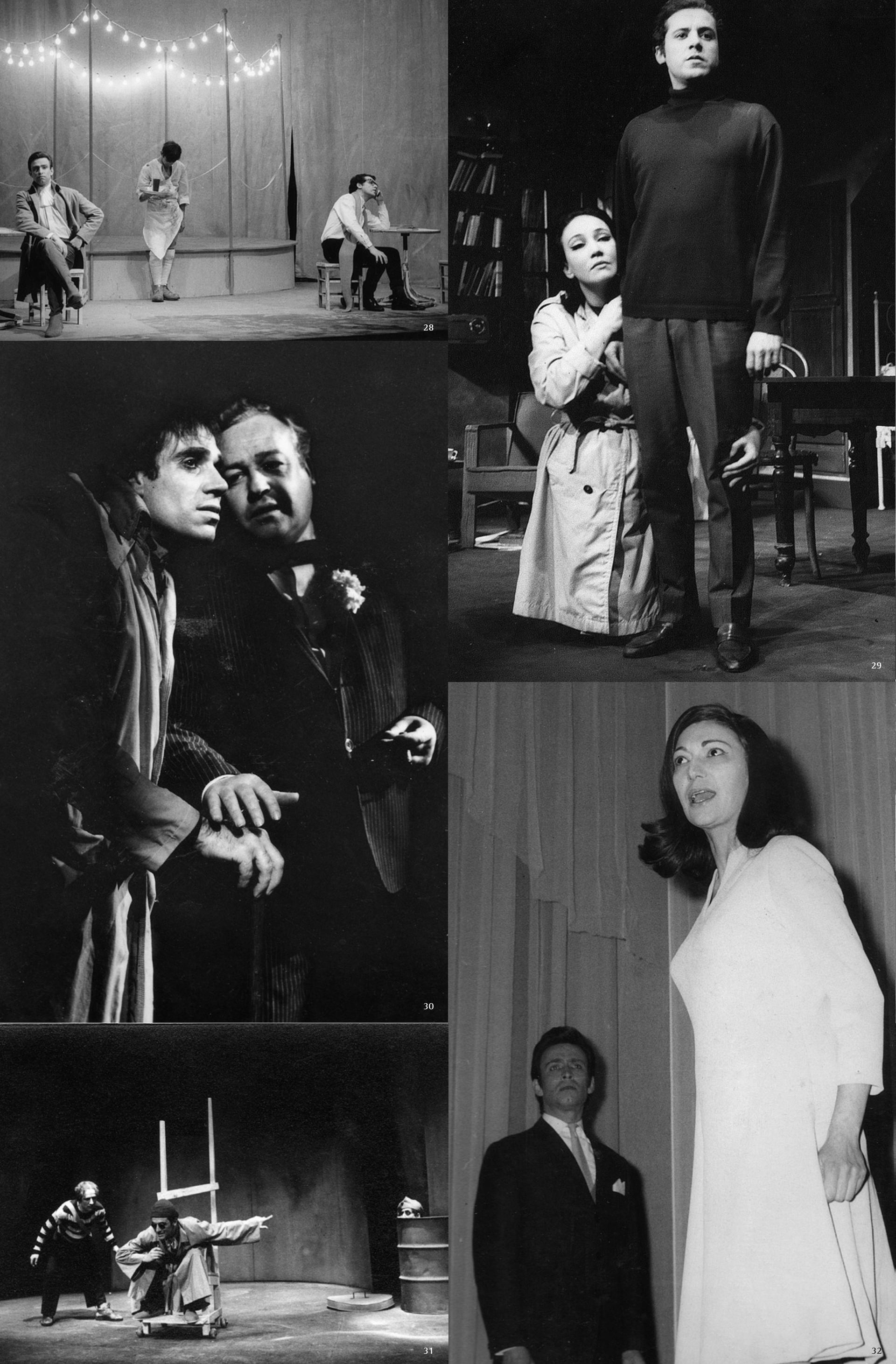



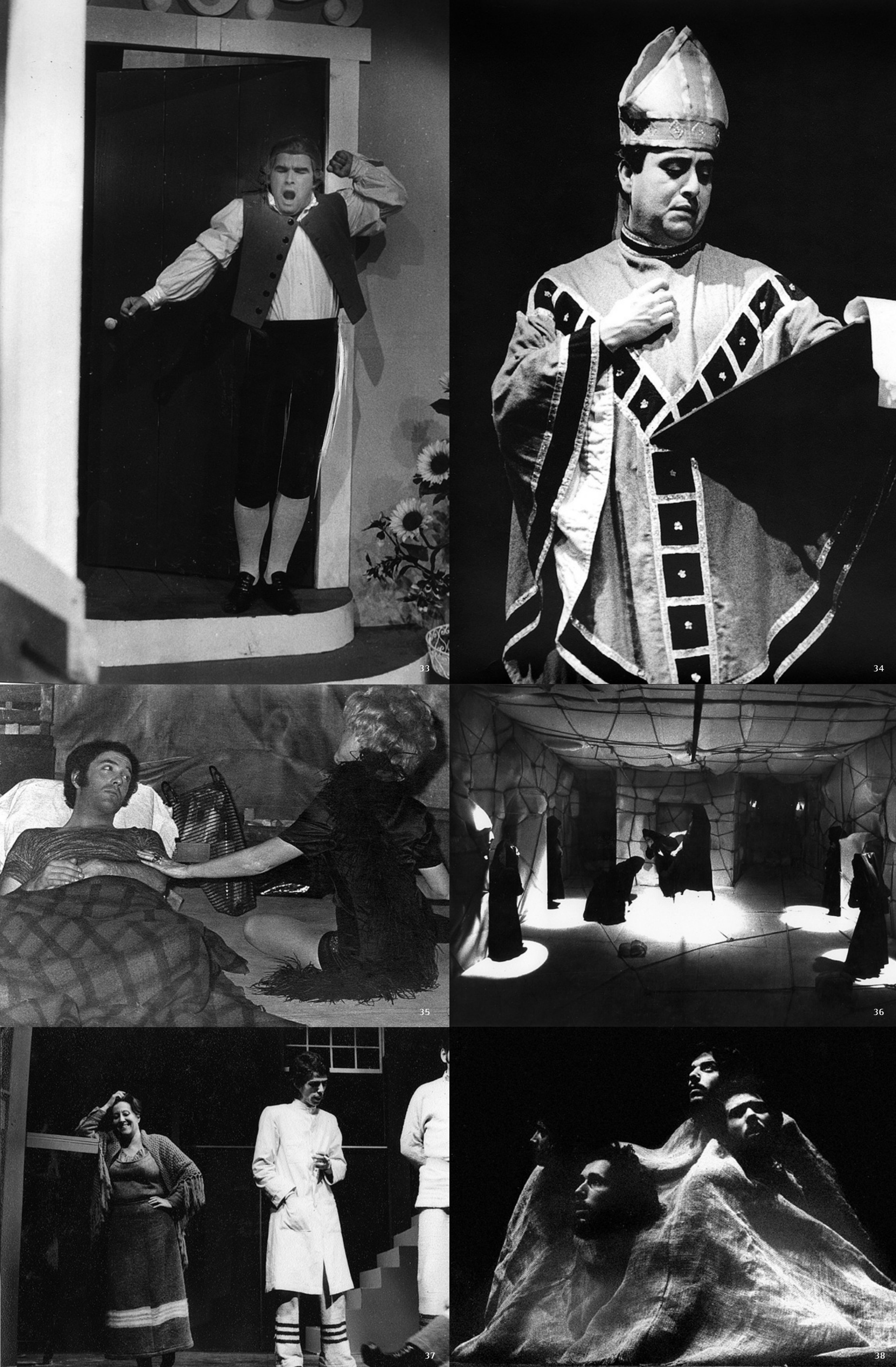


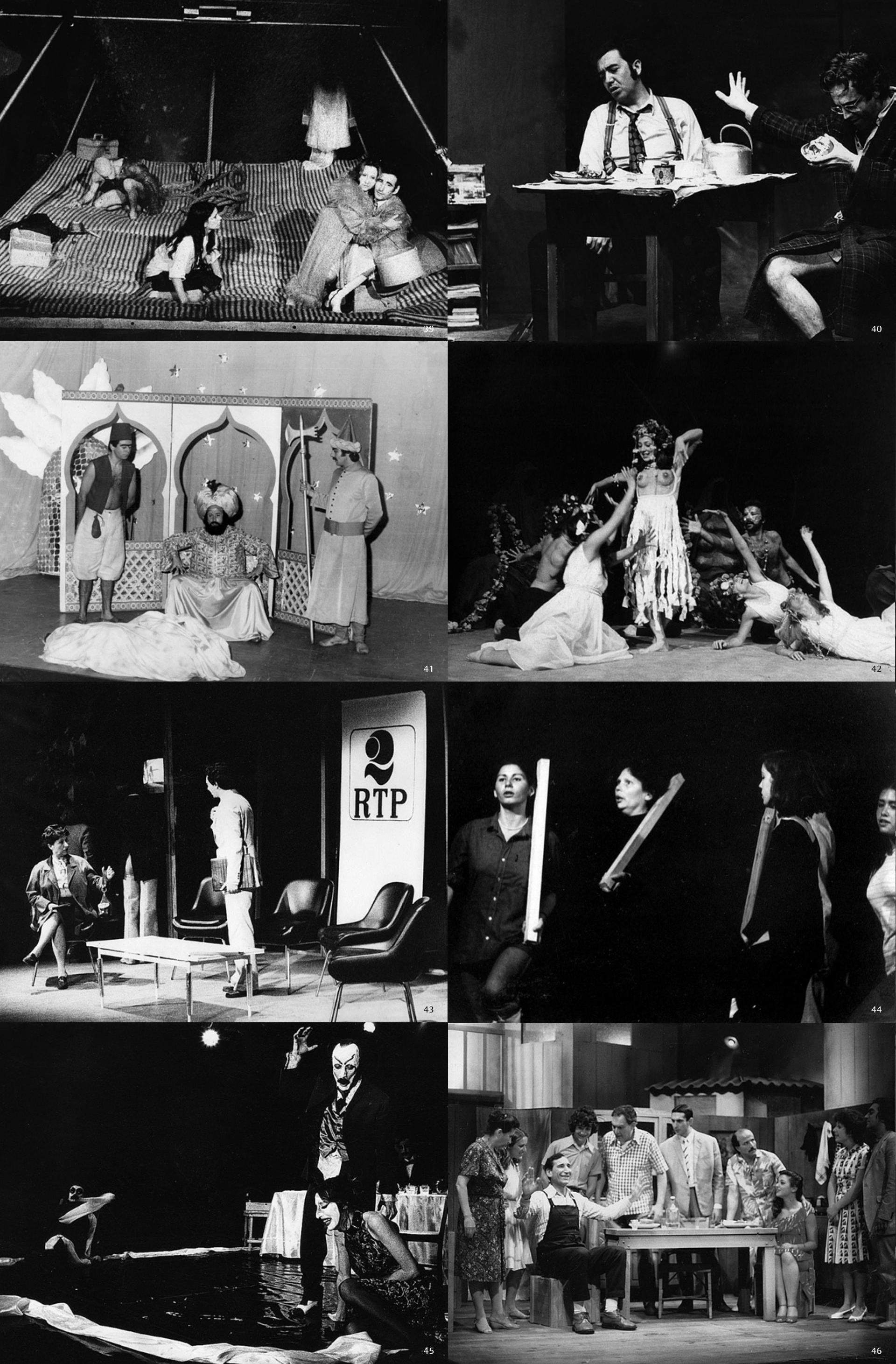




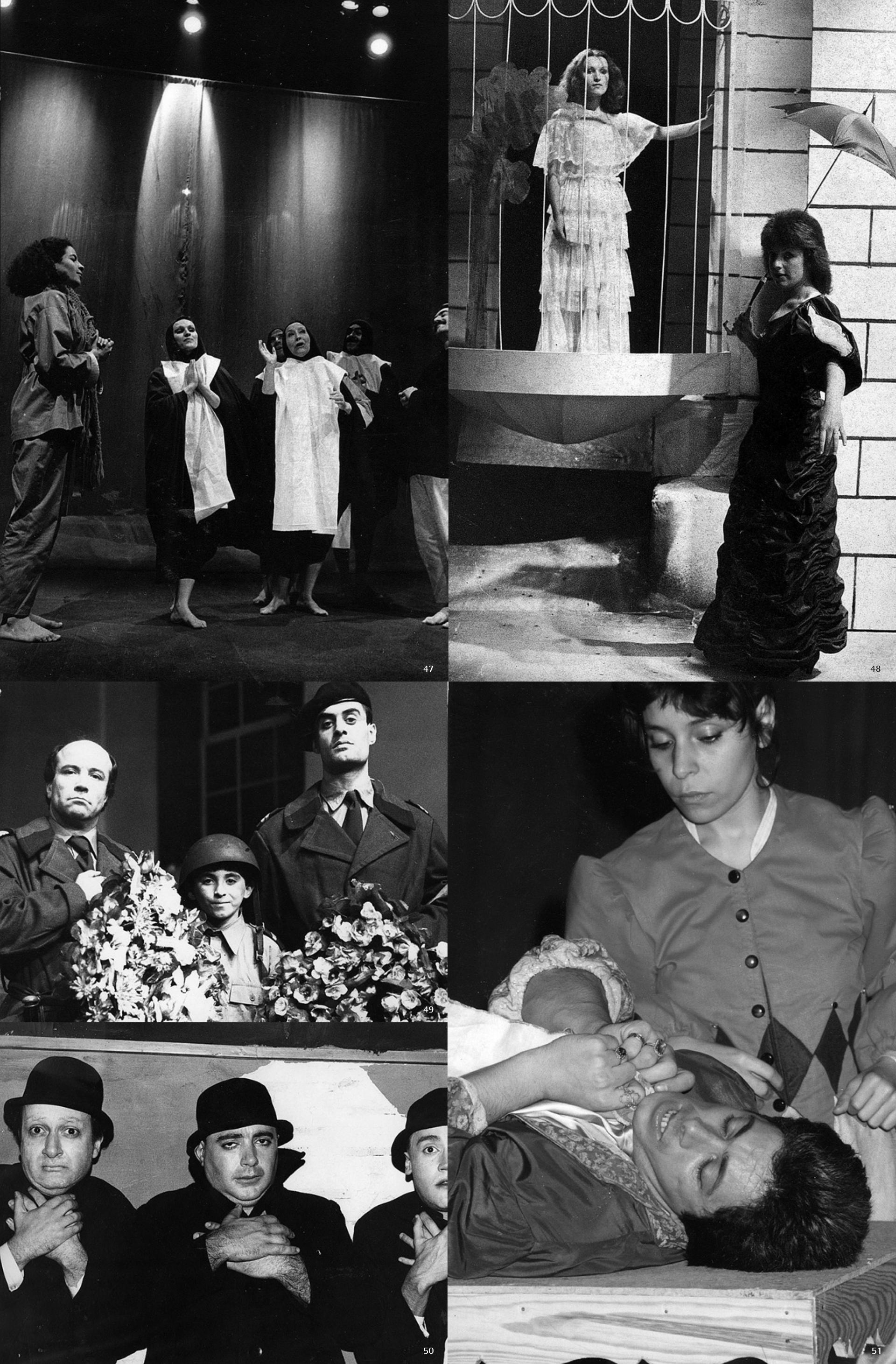



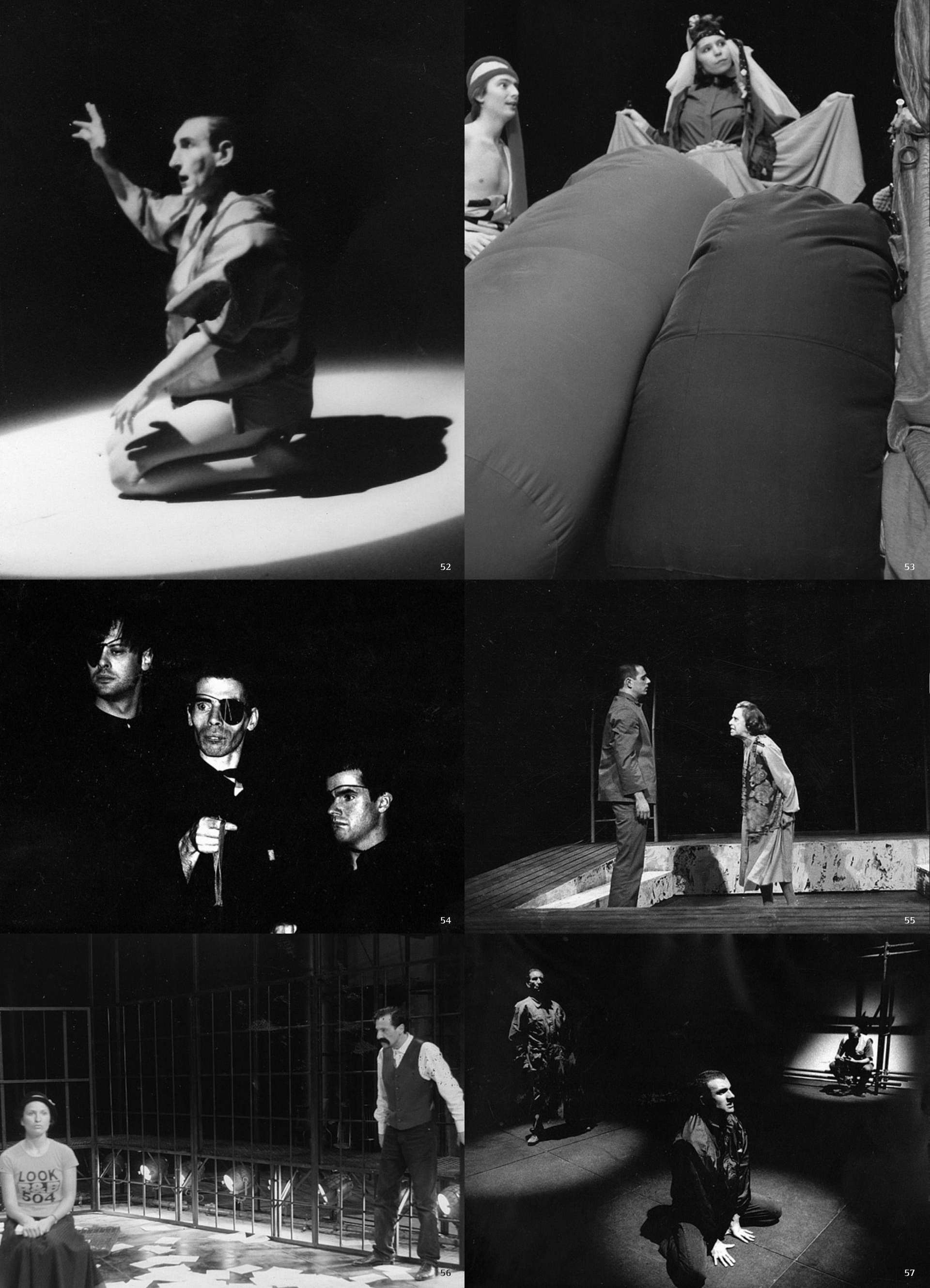

D 

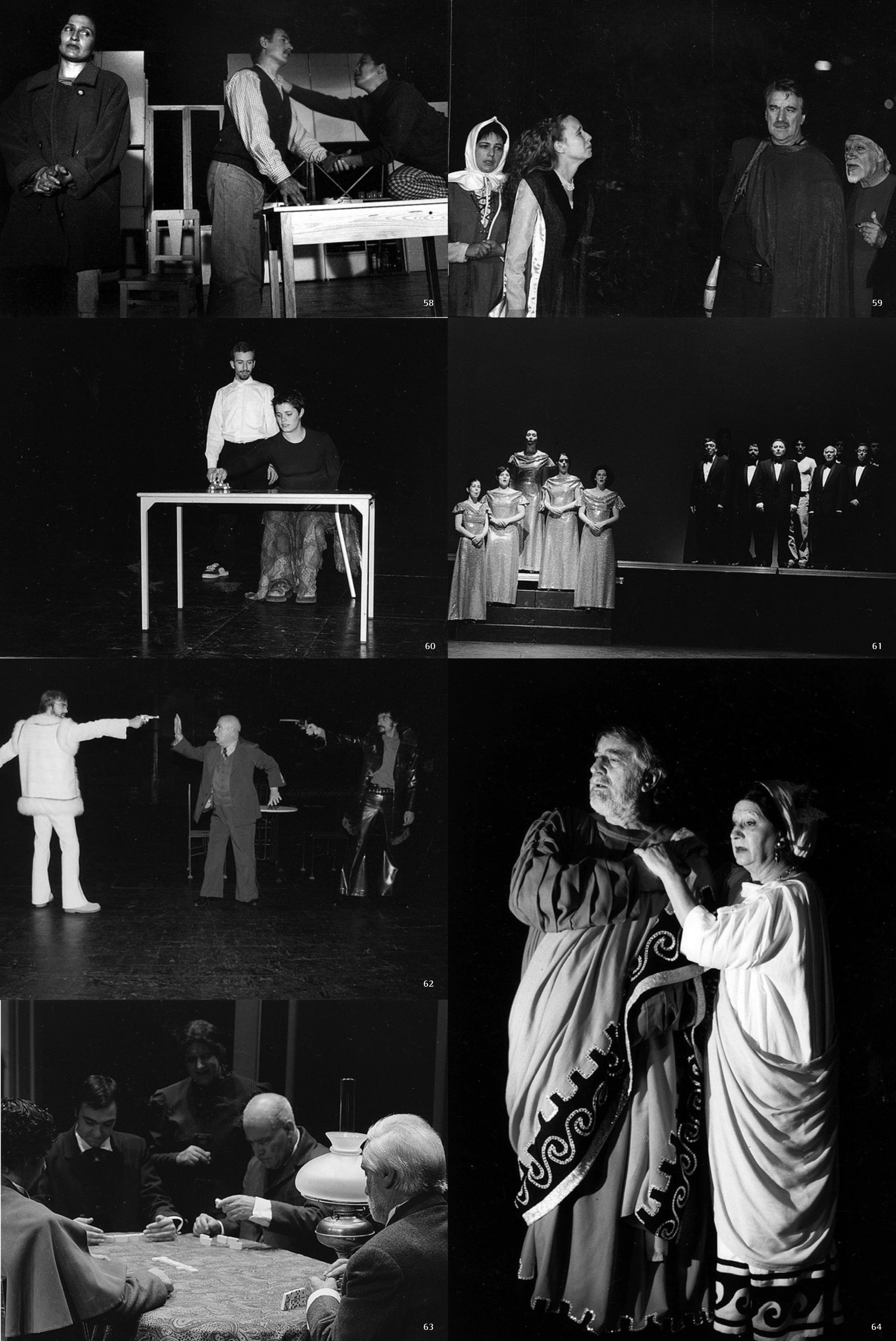


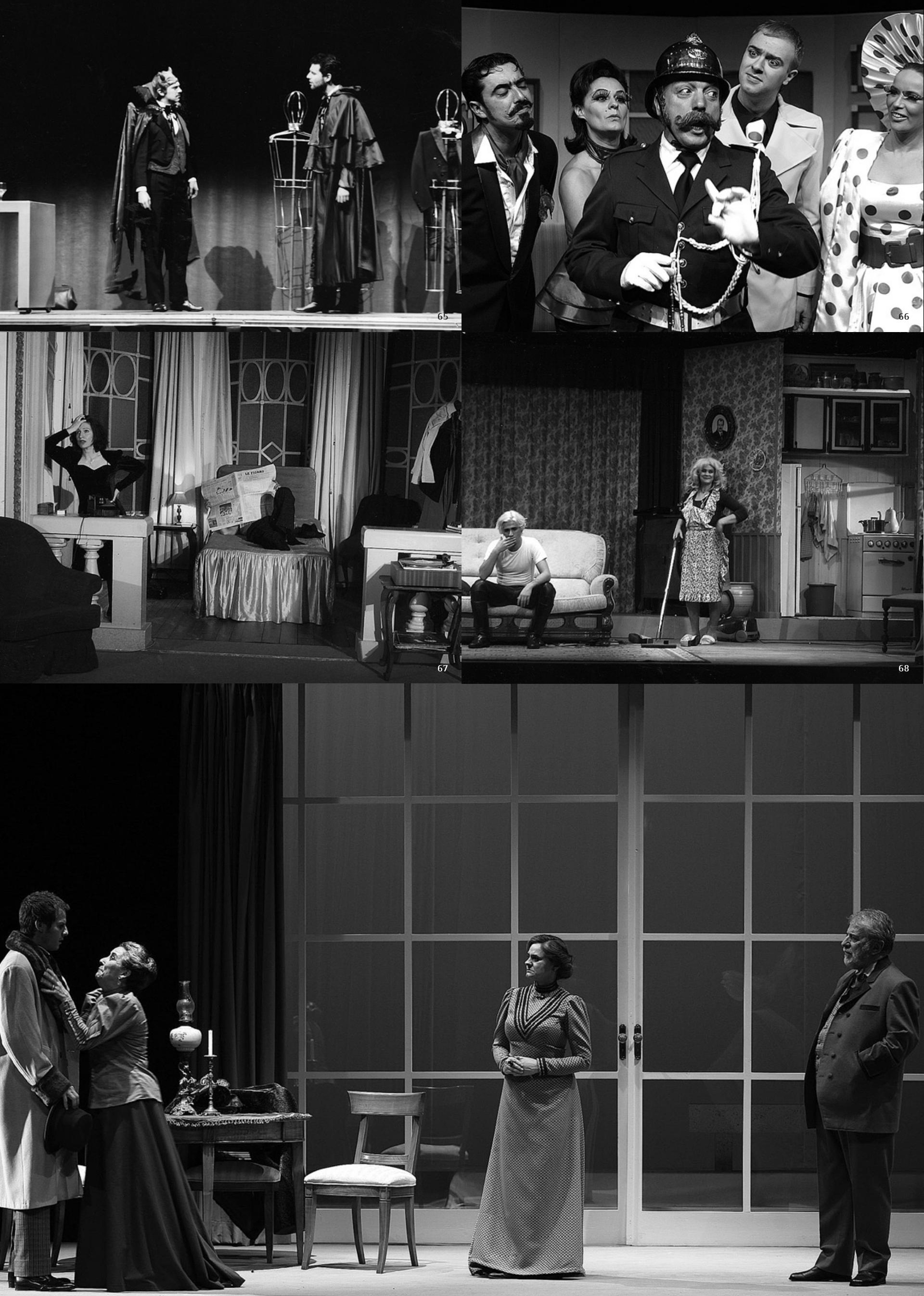



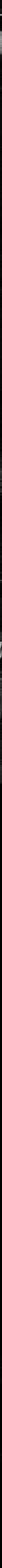